\title{
EDITORIAL
}

\section{Multifunctional Drugs As Neurotherapeutics}

An emerging paradigm that proposes the targeting of multiple components of pathobiology through a single drug molecule is gaining increasing acceptance. This "multifunctional," "multipotential," or "pluripotential" drug paradigm strongly challenges the widely held assumption that "silver bullet" agents are superior to "dirty drugs" in therapy. Although the single-target or "silver bullet" approach currently remains the major drug discovery strategy in large pharmaceutical companies, there is increasing recognition of the limitations of such an approach for complex diseases. Large-scale genomics studies have confirmed significant redundancy in proteinaceous drug targets, suggesting that highly directed drug targeting may have more limitations than a more promiscuous approach through the application of multifunctional drugs. In this issue of Neurotherapeutics, leading investigators worldwide offer new insights on the theme "multifunctional drugs." Their contributions are particularly timely as this new paradigm is fast gaining acceptance as a strategy for the treatment of complex disorders of the central nervous system (CNS).

\section{MULTIFUNCTIONAL DRUGS IN THE CNS}

Buccafusco (pp. 4-13) provides an overview of multifunctional receptor-directed drugs for disorders of the CNS. He points out that multifunctional drug candidates may be designed with a quintet of abilities that offer both palliative and disease-modifying actions; act on targets that produce additive or synergistic therapeutic responses; simultaneously evoke a therapeutic response at the desired target and prevent an undesired response mediated by an alternate target, allowing one component to promote specific druggable characteristics of the therapeutic component (such as blood-brain-barrier penetration); and prolong the duration of effectiveness of one compound by contributing to the pharmacodynamic actions of another. Examples cited provide a glimpse into the significant potential for drug discovery when new molecules are designed to act on multiple targets. This approach has the potential to enhance drug efficacy, improve dosing regimens, and reduce side effect profiles.

\section{MULTIFUNCTIONAL DRUGS FOR NEUROTRAUMA AND STROKE}

Three subsequent articles review the development and profiles of multipotential drugs in models of traumatic brain injury (TBI) or stroke. Stoica et al. explore cell cycle inhibitors and small cyclized peptides as multifunctional agents that target multiple secondary injury mechanisms in "Multifunctional Drug Treatment in Neurotrauma" (pp. 14-27). Most strategies to date have been disappointing, using drugs directed toward single pathophysiological mechanisms that contribute to early necrotic cell death. The authors describe compounds that target various secondary injury factors while simultaneously up-regulating several well established endogenous neuroprotective factors, or drugs that provide different effects across different cell types.

Vink and Nimmo (pp. 28-42) propose that the contemporary view of TBI as a heterogenous insult that varies widely in its etiology, clinical presentation, severity, and pathophysiology offers attractive opportunities for multifunctional drugs. Such drug candidates should be designed to target complex and multifaceted mechanisms that involve an interaction of acute and delayed anatomical, molecular, biochemical, and physiological events.

Treatment options for stroke remain limited despite the fact that many key pathophysiological mechanisms of cerebral ischemia have been identified in recent years. Drugs that individually target such mechanisms failed to improve clinical outcomes after stroke, likely due to a multiplicity of mechanisms involved in the cascades leading to neuronal damage after ischemia. However, drugs that target multiple etiologies of neuronal damage provided a remarkable benefit in preclinical studies. Minnerup and Schäbitz (pp. 43-52) describe the mechanisms of action of agents approved for secondary prevention of ischemic stroke, including antiplatelet, antihypertensive, and lipid-lowering drugs. These drugs exhibit considerable neuroprotective and neurorestorative properties. In addition, the candidate stroke drugs albumin, hematopoietic growth factors, and citicoline-currently in phase III clinical trials—are identified as promising multipotential agents.

\section{NEUROPSYCHIATRIC DISORDERS}

Depression has highly heterogeneous and multifactorial origins. It is unlikely that the symptoms of major depression can be efficaciously relieved by drugs that act at a single target site. Multiple-mechanism drugs may therefore offer the best hope for improved control of 
affective disorders by pharmacotherapy. Millan (pp. 5377) offers an excellent overview of this approach in the treatment of this challenging disorder.

The complex etiology of schizophrenia suggests that the symptomatology of the disorder does not arise from a single neurobiological entity, but rather may be derived from pathology at one or more receptor types. Multifactorial approaches offer real promise to the development of new therapeutics. Polypharmacy is a well utilized clinical strategy, while a multifunctional approachcalled "intramolecular polypharmacy" by Kim et al. (pp. 78-85)-may offer an even more elegant approach. Atypical antipsychotics are an already well established example of the latter. Each atypical antipsychotic possesses a unique portfolio of activities at receptors that may contribute to therapeutic effects at receptor targets that are currently thought to mediate symptoms of schizophrenia.

Silver et al. (pp. 86-93) further explore negative symptoms in schizophrenia that respond poorly to antipsychotics alone but may improve when these are augmented with selective serotonin reuptake inhibitors (SSRIs). The authors propose that the mechanism of action involves regionally selective modulation of the GABA system, pointing to evidence that indicates that the SSRI antidepressant/antipsychotic combination may be a useful paradigm to study synergistic drug interactions in schizophrenia. The discovery of definable molecular targets for synergistic SSRIantipsychotic interaction could therefore provide new directions for antischizophrenic drug development.

\section{TARGETS FOR MULTIFUNCTIONAL DRUGS}

Metabotropic glutamate receptors (mGluR) are found on cell types within and peripheral to the CNS, including neurons, microglia, astrocytes, oligodendrocytes, $\mathrm{T}$ and B lymphocytes, osteoblasts, hepatocytes, and endothelial cells. The review by Byrnes et al. (pp. 94-107) examines the multipotential therapeutic utility of mGluR modulation in acute CNS injury as well as in chronic neurodegenerative disorders. Activation of mGluR5 and group II and III mGluRs improves recovery after TBI and spinal cord injury in vivo, while antagonism of mGluR1 has similar effects, reflecting the multiple actions of these receptor subtypes. Reduction of inflammation, decreased excitotoxicity, and inhibition of both necrotic and apoptotic cell death are but a few of the potential therapeutic effects of mGluR modulation.

Neuronal expression of erythropoietin and erythropoietin receptors is up-regulated in the adult brain after injury. Erythropoietin and some of its variants, administered peripherally, cross the blood-brain barrier. Once in the brain, erythropoietin stimulates neurogenesis and neuronal differentiation and activates neurotrophic, antiapoptotic, antioxidant, and anti-inflammatory signalling that underlie its cytoprotective effects in nervous system disorders. Sirén et al. (pp. 108-127) report on the development of novel erythropoietin derivatives and erythropoietin mimetics such as asialo-erythropoietin and carbamoylated erythropoietin in preclinical studies, as well as experimental findings with recombinant human (rh)erythropoietin in clinical studies involving acute ischemic stroke, chronic schizophrenia, and chronic progressive multiple sclerosis.

\section{BIOMARKERS AS PRODROMAL DIAGNOSTICS}

Before clinical trials can be mounted ethically and efficiently, and candidate drugs moved from "bench to bedside," it is imperative that a predictive capability be in place that will allow investigators to assemble cohorts of as yet asymptomatic patients who are imminently at risk of developing symptoms in the future. Only by having access to such a cohort can neuroprotective drugs receive the same scrutiny in comparative peer-control studies that is consistently afforded other curative therapies in their clinical trial development phases. Overcoming this predicament is particularly crucial for the development of multifunctional drugs. At present, clinical diagnosis of many neurodegenerative diseases, including Alzheimer's disease (AD) and Parkinson's disease (PD), is based on a constellation of symptoms and manifestations. The development of reliable biomarkers will not only facilitate early disease diagnosis but also assist in identifying pathological mechanisms more definitively and reliably than traditional cognitive and behavioral phenotypes that are currently in use. Halperin et al. (pp. 128-140) review biochemical, genetic, and neuroimaging biomarkers and discuss their predictive value as indicative for disease vulnerability to detect subgroups at risk for PD and AD. Such biomarkers would provide important tools to determine the clinical efficacy of novel neuroprotective drug candidates.

\section{MULTIFUNCTIONAL DRUGS FOR PD AND AD}

Several reviews focus on diverse groups of drug candidates with varying degrees of multifunctionality and a diverse array of drug targets in PD and AD. Petzer et al. (pp. 141-151) summarize recent efforts to develop dualacting monoamine oxidase $\mathrm{B}$ (MAO-B) inhibitors and adenosine $A_{2 A}$ antagonists by using caffeine as a lead compound. Antagonists of the adenosine $\mathrm{A}_{2 \mathrm{~A}}$ receptor are considered promising agents for the symptomatic treatment of PD and may also have neuroprotective properties. MAO-B inhibitors may also possess neuroprotective properties in part by reducing the damaging effect of dopamine turnover in the brain, and by attenuating pro- 
apototic cascades in the brain. Based on these observations, multifunctional compounds that inhibit MAO-B and antagonize $\mathrm{A}_{2 \mathrm{~A}}$ receptors simultaneously may have significant utility in the management of PD.

Memoquin is a new drug candidate specifically designed and synthesized in a deliberate attempt to develop a multitarget-directed ligand able to interfere with different key target points of $\mathrm{AD}$ neurodegeneration. In aged mice, memoquin ameliorates the cholinergic deficit and behavioral impairment, the expression and deposition of $\mathrm{A} \beta$, and the hyperphosphorylation and deposition of tau protein at different stages of the neurodegenerative process. Bolognesi et al. (pp. 152-162) report that the promising in vitro and in vivo biological profile of memoquin demonstrates the suitability of a multifunctional drug design strategy to obtain innovative drug candidates for the treatment of neurodegenerative diseases, in particular AD.

\section{NEW CHEMICAL SCAFFOLDS FOR BRAIN-DIRECTED MULTIFUNCTIONAL DRUGS}

Weinreb et al. (pp. 163-174) describe their drug discovery strategy of combining the neuroprotective/neurorestorative anti-parkinsonian drug and selective MAO-B inhibitor rasagiline with rivastigmine (a compound with cholinesterase inhibitory activity) to produce a new multifunctional drug, ladostigil. A second derivative of rasagiline, M-30, was developed by amalgamating the propargyl moiety of rasagiline into the skeleton of the novel brain permeable neuroprotective iron chelator VK-28. The authors discuss the multimodal effects of these two rasagilinecontaining hybrid molecules in vivo and in vitro, and show that these new drugs act by a variety of mechanisms, including the regulation of amyloid precursor protein processing, activation of protein kinase $\mathrm{C}$ and mitogen-activated protein kinase signaling pathways, inhibition of cell death signaling, and up-regulation of neurotrophic factors.

Polycyclic ring structures have been found to be exceedingly useful as starting scaffolds in medicinal chemistry programs to develop multifunctional drugs, and may also be useful moieties that can be added to existing structures to improve the pharmacokinetic properties of drugs currently used in the clinic or under development. Van der Schyf and Geldenhuys (pp. 175-186) review research using polycyclic compounds as substrate molecules to develop multifunctional drugs. Pentacyclounde- cylamine derivatives block both the neuronal L-type calcium channel and $N$-methyl-D-aspartate receptors in vitro, are neuroprotective in the 1-methyl-4-phenyl1,2,3,6-tetrahydropyridine model of PD, and attenuate brain edema and infarct size in both the permanent and transient middle cerebral artery occlusion model of stroke. Other polycyclic ring structures derived from the triquinane structure show similar in vitro properties, suggesting that these compounds also may interact with both voltage-gated calcium channels as well as the NMDA receptor.

Li et al. (pp. 187-201) describe the development of bis(7)-cognitin. This unique compound acts through a multitude of mechanisms, including concurrent inhibition of AChE, the NMDA receptor, nitric oxide synthase, and amyloid precursor protein/ $\beta$-amyloid cascade, imparting the compound with significant neuroprotective activity in models of AD.

\section{SUMMARY}

In summary, the rapidly expanding interest in multifunctional drugs as an approach to the treatment of neurological disorders will create exciting new opportunities for the future development of novel neurotherapeutics. If robust prodromal diagnostic methodologies for the most common neurodegenerative diseases can be developed in parallel with the emergence of an array of multimodal drugs that target a reasonable selection of pathological etiologies, it is likely that significant strides will be made in the treatment of, or-even more desirable a goal- the prevention of the most common and burdensome neurological disorders.

Cornelis J. Van der Schyf, D.Sc. Chair and Professor of Pharmaceutical Sciences Professor of Neurobiology Northeastern Ohio Universities Colleges of Medicine and Pharmacy Rootstown, Ohio

Moussa B. H. Youdim, Ph.D. Finkelstein Professor of Life Sciences Professor of Pharmacology Director of Eve Topf Centre of Excellence for Neurodegenerative Diseases Technion-Rappaport Faculty of Medicine Haifa, Israel 